\title{
Massive stellar content of the Galactic supershell GSH 305+01-24 ${ }^{\star}$
}

\author{
N. T. Kaltcheva ${ }^{1}$, V. K. Golev ${ }^{2}$, and K. Moran ${ }^{1}$ \\ 1 Department of Physics and Astronomy, University of Wisconsin Oshkosh, 800 Algoma Blvd., Oshkosh, WI 54901, USA \\ e-mail: kaltchev@uwosh.edu \\ 2 Department of Astronomy, Faculty of Physics, St Kliment Ohridski University of Sofia, 5 James Bourchier Blvd., 1164 Sofia, \\ Bulgaria \\ e-mail: valgol@phys.uni-sofia.bg
}

Received 12 March 2013 / Accepted 2 December 2013

\section{ABSTRACT}

\begin{abstract}
Aims. The distribution of $\mathrm{OB}$ stars along with $\mathrm{H} \alpha,{ }^{12} \mathrm{CO}$, dust infrared emission, and neutral hydrogen is carried out to provide a more complete picture of interactions of the young massive stars and the observed supershell GSH 305+01-24.

Methods. The studied field is located between $299^{\circ} \leq l \leq 311^{\circ}$ and $-5^{\circ} \leq b \leq 7^{\circ}$. The investigation is based on nearly 700 O-B9 stars with currently available $u v b y \beta$ photometry. The derived stellar physical parameters were used to establish a homogeneous scale for the distances and extinction of light for major apparent groups and layers of foreground and background stars in Centaurus and to study the interaction with the surrounding interstellar medium.

Results. The distance to the entire Centaurus star-forming complex is revised, and a maximum of the OB-star distance distribution is found at $1.8 \pm 0.4$ (rms) kpc. The massive star component of GSH 305+01-24 is identified at about 85-90\% completeness up to 11.5-12 mag. The projected coincidence of the OB stars with the shell and the similarities between the shell's morphology and the OB-star distribution indicate a strong interaction of the stellar winds with the superbubble material. We demonstrate that these stars contribute enough wind injection energy to explain the observed size and expansion velocity of the supershell. The derived stellar ages suggest an age gradient over the Coalsack Loop. Continuous star formation might be taking place within the shell with the youngest stars located toward its periphery, and the open cluster NGC 4755 is the oldest. A layer of very young stars at $1 \mathrm{kpc}$ is detected, and its connection to both GSH 305+01-24 and the foreground GSH 304-00-12 H I shells is investigated.
\end{abstract}

Key words. open clusters and associations: individual: Centaurus star-forming field - ISM: individual objects: GSH 305+01-24 stars: early-type

\section{Introduction}

The Cen OB1 association represents one of the prominent starforming regions of the Milky Way. It forms part of an extended star-formation complex in the disk between Galactic longitudes $299^{\circ}$ and $311^{\circ}$. The field is dominated by the H I supershell GSH 305+01-24 (McClure-Griffiths et al. 2001) and its optical counterpart, the Coalsack Loop, which was first reported by Walker \& Zealey (1998). In both two-armed and four-armed models of the Galaxy, the field is associated with the CarinaSagittarius arm or with an inner spur of the arm (see for example Humphreys \& Kerr 1974). The field is located in the direction of the Lower Centaurus-Crux (LCC) association, which is one of the most prominent tracers of the Gould Belt and is likely to be our closest giant star-forming complex (Comerón 2001), which could also represent a large-scale complex in the region of the Orion Arm (Sartori et al. 2003; de Zeeuw et al. 1999; Elias et al. 2009).

The direction between Galactic longitudes $302^{\circ}$ and $313^{\circ}$ is tangent to the Scutum-Crux arm (also referred to as the Scutum-Centaurus, or Centaurus arm), corresponding to maxima in the thermal radio continuum, $\mathrm{HI}$ and $\mathrm{CO}$ emissions (Taylor \& Cordes 1993; Bloemen et al. 1990; Bronfman 1992; Benjamin 2008). As one of the major Galactic star-forming

* Tables 1 and 3 are only available at the CDS via anonymous ftp to cdsarc.u-strasbg.fr (130.79.128.5) or via

http://cdsarc.u-strasbg.fr/viz-bin/qcat?J/A+A/562/A69 regions, Centaurus can be used to model and/or test various spiral morphologies from grand-design logarithmic spirals (Russeil 2003; Vallée 2008) to designs dominated by rings or pseudorings (see Rautiainen \& Mel'nik 2010 for a recent discussion). The Centaurus field may be particularly important for understanding a spiral design with two principal arms and the underlying enhancements in the old stellar disk, which has recently been discussed by Lépine et al. (2001) and Benjamin (2008).

There have been several attempts to establish the distance to Cen OB1 (Humphreys 1978; Blaha \& Humphreys 1989; Garmany \& Stencel 1992; Mel'nik \& Efremov 1995), most of which were based on the photometric data of Blaha \& Humphreys (1989) or on earlier studies. All estimates used $U B V$ photometry and spectrophotometric calibrations and placed the association 1.9 to $2.5 \mathrm{kpc}$ from the Sun. Recently, using proper motion and spectrophotometric distances, Corti et al. (2012) have compiled a list of 56 members of the association and find an average distance of $2.6 \pm 0.4 \mathrm{kpc}$.

In the present study we combine surveys of $\mathrm{H} \alpha,{ }^{12} \mathrm{CO}$, dust infrared emission at $100 \mu \mathrm{m}$, and neutral hydrogen with intermediate-band $u v b y \beta$ photometry to examine the correlation between the location of the $\mathrm{OB}$ stars and the neutral and ionized material in the GSH 305+01-24 supershell. Based on homogeneous distances of nearly 700 early-type stars, we select spatially coherent stellar groupings and revise the classical concept of the Cen OB1 association. 


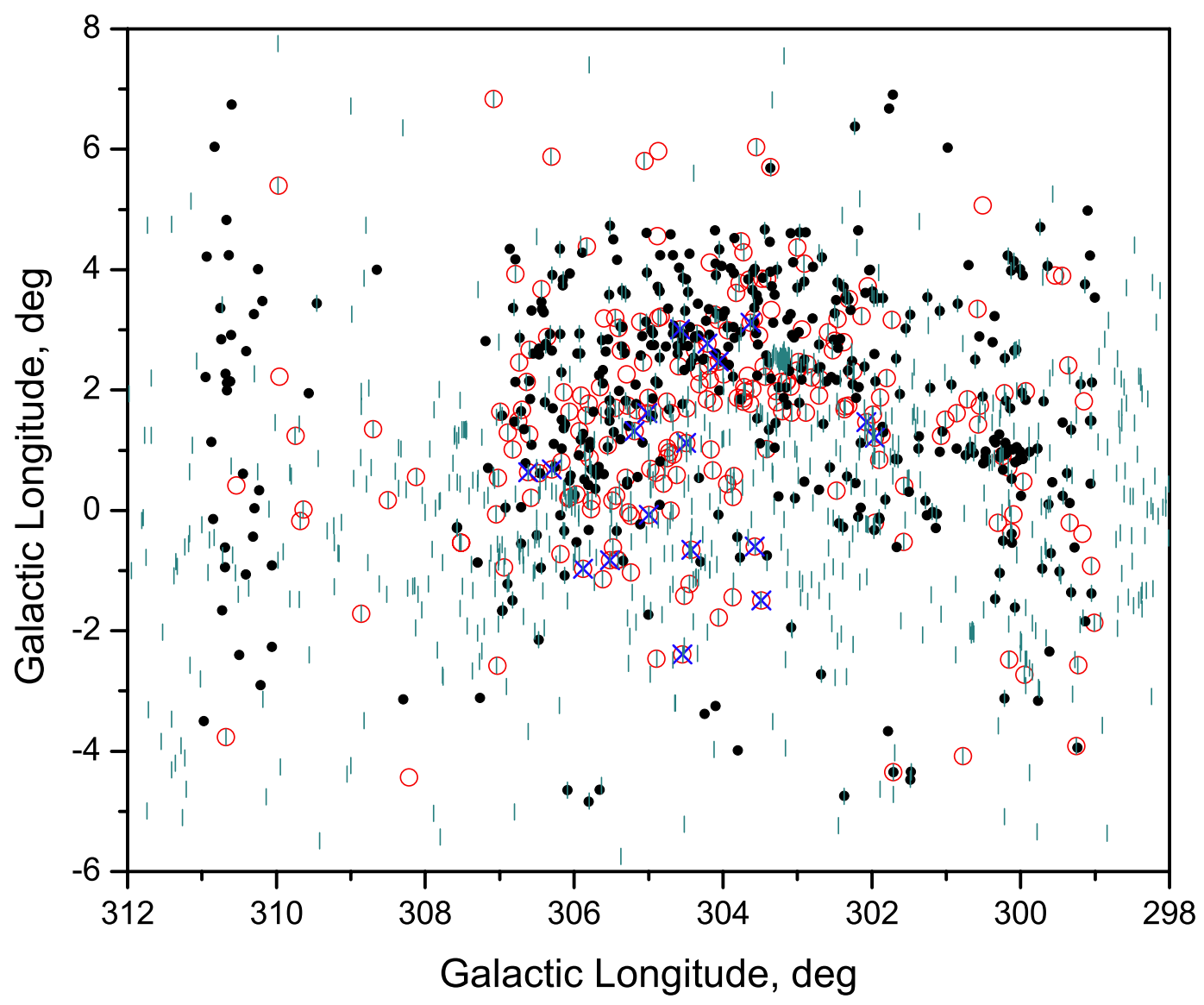

Fig. 1. The sample of 693 stars plotted in Galactic coordinates. Intrinsically bright stars with $M_{V}<-2$ mag (which are also located farther than $1 \mathrm{kpc}$ ) are marked by red open circles and stars intrinsically fainter than $-2 \mathrm{mag}$ (which are all found closer than $1 \mathrm{kpc}$ ) by black filled circles. Stars from the classical Cen OB1 association are marked by blue $\times$-symbols. The stars from the Luminous Stars catalog (Reed 2003) are represented by green vertical symbols. The concentrations represent NGC $4755\left(l, b=303^{\circ} .206,2.59\right)$ and St $16\left(l, b=306^{\circ} .15\right.$, 0.0656). See details in Sect. 3 . See the electronic edition for a color version of the figure.

This study is organized as follows. In Sects. 2 and 3 we discuss the stellar sample and findings regarding groupings and the overall distribution of the O-B9 stars toward Centaurus. Section 4 is focused on the census of OB stars within GSH 305+01-24 and on the correlation between massive stars and interstellar material. Brief concluding remarks are presented in Sect. 5 .

\section{The stellar sample}

Among the wide variety of photometric systems available today, the $u v b y \beta$ photometry is arguably better suited for studying individual stars in terms of stellar luminosities and distance moduli than any other photometric system in wide use. A detailed discussion of the $u v b y \beta$ system can be found in Strömgren (1966). In the O-B9 spectral range considered in this study, the $\beta$ index is a measure of the strength of the $\mathrm{H} \beta$ line and thus a luminosity indicator that is primary used to calculate $M_{V}$. The $(b-y)$ index is a temperature indicator, providing the true stellar color, hence the interstellar reddening. The color difference $c_{1}=(u-v)-(v-b)$ is a measure of the Balmer discontinuity and an indicator of the effective temperature in O-B9 stars, which is also used in the $M_{V}$ calculation. The color difference $m_{1}=(v-b)-(b-y)$ measures the blocking by metallic lines, but it is not used in any of the calibrations applicable to O-B9 stars. A recent description of the $u v b y \beta$ photometric quantities is provided by Árnadóttir et al. (2010).
The sample for the present study contains 693 (mostly) field stars within the coordinate range $299^{\circ} \leq l \leq 311^{\circ}$ and $-5^{\circ} \leq b \leq+7^{\circ}$. The stars are of spectral type $\mathrm{O}$ to $\mathrm{B} 9$ and have complete $u v b y \beta$ photometry. Some of the brightest stars of several loose clusters, such as St 16, are included in the sample, as listed in Table 1, which is only available electronically. In addition, 56 members of the young open cluster NGC 4755 ( $\kappa$ Crucis cluster, Jewel Box) are considered. All uvby $\beta$ photometry was extracted from the catalog of Hauck \& Mermilliod (1998). The homogeneity of similar samples is discussed in earlier papers that studied the structure of Galactic star-forming regions (see for example Kaltcheva \& Golev 2012).

\subsection{Completeness of the stellar sample}

The locations of the 693 sample stars in Galactic coordinates are shown in Fig. 1. When selecting the stars we considered a larger field than the projected size of GSH 305+01-24. This was done with the purpose of selecting all massive stars projected towards the supershell and its environments, i.e. the entire Centaurus field. Figure 1 shows the 693 sample stars that are marked with two different symbols, according to the separation we establish in Sect. 3. There we separate the sample into those stars closer than $1 \mathrm{kpc}$ (which are intrinsically fainter than $M_{V}$ of -2 mag) and those stars which are more distant and intrinsically brighter than -2 mag. 
The best possible way to estimate the sample completeness is to compare to the Luminous Stars (LS) Catalog (see Stephenson \& Sanduleak 1971; Reed 2003). In the coordinate range $l=\left(299^{\circ}, 311^{\circ}\right), b=\left(-5^{\circ}, 7^{\circ}\right), 962 \mathrm{LS}$ are found (Fig. 1). Of these, 172 correspond to intrinsically fainter stars and 180 to intrinsically brighter ones according to our separation criterion (see Sect. 3). This implies that the LS catalog contains stars that are intrinsically faint and possibly not connected to the starforming field.

To estimate the completeness of the stellar sample projected toward the GSH 305+01-24 supershell, we restrict the coordinate range to $l=\left(300^{\circ}, 307^{\circ}\right), b=\left(-5^{\circ}, 7^{\circ}\right)$. If NGC 4755 is excluded, there are $675 \mathrm{LS}$ stars in this region. Of them 152 correspond to sample stars intrinsically fainter than $M_{V}=-2 \mathrm{mag}$ (393 in total) and 158 to intrinsically brighter stars (202 in total). It seems reasonable to assume that the same proportionality between the number of stars that are brighter and fainter than -2 mag is true for all 675 LS stars projected toward the shell. This would imply that the sample located beyond $1 \mathrm{kpc}$ is at least $70 \%$ to $80 \%$ complete to the limit of the LS catalog. In addition, our sample contains nearly 50 intrinsically bright distant stars not identified as LS stars, which should improve its completeness.

One may note that there are not many $u v b y \beta$ data toward the longitude interval $307^{\circ}$ to $310^{\circ}$ in Fig. 1. This might indicate incompleteness because the Hauck \& Mermilliod (1998) catalog is a compilation from different sources with different completeness levels depending on the position on the sky. On the other hand, the LS catalog that is complete to $m_{\mathrm{ph}} \sim 12$ limit to some extent shows similar behavior, suggesting the absence of prominent star formation in this direction. In any case, this region is outside the cavity of the H I and H II shells, so it should not affect our findings below.

\subsection{The interstellar extinction and distances}

The procedure used to obtain color excesses and distances for sample stars is described in detail in Kaltcheva \& Hilditch (2000). The color excesses for luminosity class (hereafter LC) III, IV, and V were obtained via Crawford's 1978 calibration. The calibration by Kilkenny \& Whittet (1985) was used for LC II, Ib, Iab, and Ia. The relations $R=3.2$ and $E(B-V)=E(b-y) / 0.74$ were adopted to obtain $V_{0}$. The calibration by Balona \& Shobbrook (1984) was utilized for all O-B9 stars to derive values of $M_{V}$. Since the stars are of early spectral type, the presence of emission lines in their spectra is the largest source of error in the calculated absolute magnitudes. However, the $\beta$ vs. $c_{0}$ diagram (not shown here) indicates that only seven stars in the sample deviate from the main sequence or have photometry affected by emission. For all stars with observed $\beta$ lying outside the limits of the $M_{V}\left(\beta, c_{0}\right)$ calibration and for all known emission-line stars, $\beta$ was calculated from $c_{0}$ (see for details Balona 1994; and Kaltcheva \& Hilditch 2000). Such a procedure yields a distance in excellent agreement with the recalculated HIPPARCOS data (see for example Kaltcheva 2009; and Kaltcheva \& Makarov 2007).

The photometric data and the derived stellar parameters are summarized in Table 1 followed by the SIMBAD identifier, Galactic coordinates, $u v b y \beta$ photometric data, MK type, dereddened photometry, color excess, calculated absolute magnitude, and true distance modulus. The uncertainties in $M_{V}$ are on the order of \pm 0.3 mag for O and B types of LC III-V and \pm 0.5 mag for B-type supergiants (Balona \& Shobbrook 1984). An uncertainty of $\pm 0.3 \mathrm{mag}$ in $M_{V}$ propagates to an asymmetric error of $-13 \%$ to $+15 \%$, and uncertainties of \pm 0.5 mag result in $-21 \%$ to $+26 \%$ error in the derived distances.

Since the photometry used in this paper comes from different sources, the homogeneity of the sample is an important issue. The above-mentioned errors include the possible systematic errors in the existing photometric data. Comparisons of existing uvby $\beta$ datasets collected by various authors are generally in a good agreement (see Kaltcheva et al. 2000). The estimated uncertainty in the calculated stellar distances due to possible systematic deviations could not exceed $3-5 \%$. A similar approach has been followed by Torra et al. (2000) who also use the $u v b y \beta$ photometry from Hauck \& Mermilliod (1998) to derive individual distances and age in their study of the local kinematics of young stars. Depending on the spectral type and luminosity class, the errors estimated by Torra et al. (2000) range between $14 \%$ and $23 \%$. It should be noted that in the $u v b y \beta$ system both the color excess and absolute magnitude calculations do not rely on a precise determination of spectral sub-type, since the calculations are carried out in the same manner for types from $\mathrm{O}$ to B9, depending on LC only for the color excess derivation.

To infer the physical stellar parameters from the photometry, the spectral and luminosity classifications were extracted from the SIMBAD database and checked against the classification from the $\left[c_{1}\right]$ vs. $\left[m_{1}\right]$ diagram (not shown here). Only three stars showing disagreement between the photometric quantification and SIMBAD MKK types were removed from the sample. Great care was taken to resolve all cases of suspected luminosity class misclassification, because different calibrations are used to calculate the color excesses for different LC types. For stars of LC types I and II we compared the LC accepted in SIMBAD to all of the literature sources of luminosity classification, as well as to the photometric quantification, and we did not find any disagreements.

The Cen OB1 association was previously studied in the uvby $\beta$ system by Kaltcheva \& Georgiev (1994) using all 28 early-type members identified by Humphreys (1978). The present work offers several improvements to that study. Many more stars are included here, utilizing homogenized $u v b y \beta$ photometry from the Hauck \& Mermilliod (1998) catalog. New MKK classifications made available in the past decade permit a refinement of the LC classification for a number of stars. Most important, the $M_{V}$ calibration of Balona \& Shobbrook (1984) is applied to all stars, instead of the Zhang (1983) calibration used by Kaltcheva \& Georgiev (1994) for stars of LC I and II. The Balona \& Shobbrook (1984) calibration has been tested extensively using HIPPARcos data and is applicable to evolved stars as well as dwarfs. Our extensive tests of both calibrations show that the relations of Zhang (1983) may overestimate brightness for LC I and II stars. Since the majority of members identified by Humphreys (1978) are giants and supergiants, such a change significantly affects the average distance to the association.

Including all photometric and spectral classification data available at present helps to resolve cases of stars with controversial LC. For example, HD 114122 has four spectral classifications in SIMBAD: O+, B1Iab, B0III, and B0.5Ia-ab (the last adopted by Humphreys 1978 and by Kaltcheva \& Georgiev 1994). An inspection of the star's location in the $\left[c_{1}\right] /\left[m_{1}\right]$ and $\beta /[u-b]$ and $\beta /\left[c_{1}\right]$ diagrams (not shown here) indicates LC II-III. Table 2 presents a summary of color excess $E(b-y)$ and $M_{V}$ calculations for this star for different LCs with the $M_{V}$ calibrations noted above (Zhang 1983; and Balona \& Shobbrook 1984). It can be noted that the DM differs by only 0.2 mag between LC V-III and LC Ia for the Balona \& Shobbrook (1984) calibration (for the O-B1 spectral range $M_{V}$ is almost the same 

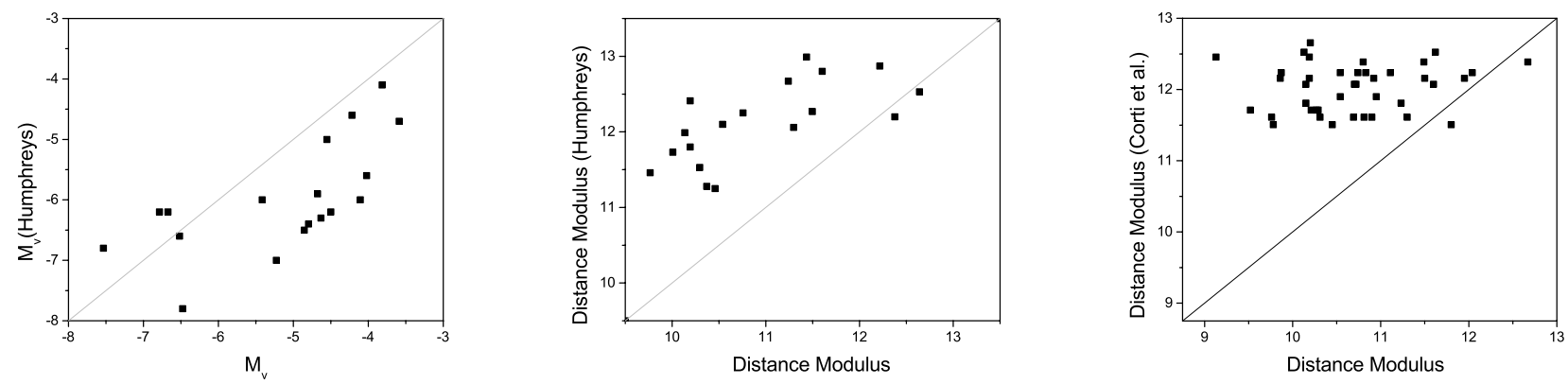

Fig. 2. Comparison of $u v b y \beta$ absolute magnitudes and distance moduli obtained here (plotted on the abscissa) with previously published spectroscopic absolute magnitudes and distance moduli.

Table 2. Color excess and distance modulus as a function of luminosity class (LC) obtained via the $M_{V}$ calibrations of Zhang (1983) and Balona \& Shobbrook (1984) for HD 114122 as an example.

\begin{tabular}{lccccccccc}
\hline \hline LC & $\beta$ & $(b-y)_{0}$ & $c_{0}$ & $E(b-y)$ & $V_{0}$ & $M_{V}$ (Zhang) & DM (Zhang) & $M_{V}(\mathrm{~B} \& S)$ & DM (B\&S) \\
\hline V-III & 2.564 & -0.127 & -0.107 & 0.641 & 5.824 & -5.414 & 11.238 & -5.41 & 11.24 \\
II & 2.564 & -0.127 & -0.107 & 0.641 & 5.824 & -5.845 & 11.669 & -5.41 & 11.24 \\
Ib & 2.564 & -0.096 & -0.101 & 0.61 & 5.958 & -5.862 & 11.82 & -5.38 & 11.34 \\
Iab & 2.564 & -0.069 & -0.096 & 0.583 & 6.073 & -5.875 & 11.948 & -5.36 & 11.43 \\
Ia & 2.564 & -0.053 & -0.093 & 0.567 & 6.143 & -5.884 & 12.026 & -5.34 & 11.48 \\
\hline
\end{tabular}

for all LC). The calibration of Zhang (1983) provides intrinsically brighter $M_{V}$ in the case of LC I and II. In her study, Humphreys (1978) lists $M_{V}=-6$ and $\mathrm{DM}=12.67\left(A_{V}=2.61\right)$. Our calculations yield a slightly larger $M_{V}$ (regardless of $M_{V}$ calibration used) and a smaller DM, respectively (DM = $11.24 \mathrm{mag}$ was adopted here). A similar analysis was performed for all stars in the sample with controversial LC designations.

Careful consideration of LC classification for sample stars and use of a more appropriate $M_{V}$ calibration result in better distance determinations, thereby helping to distinguish between closely spaced groups lying along the line of sight.

\section{Distribution of O-B9 stars toward Centaurus}

Humphreys (1978) lists 29 stars as members of Cen OB1 at $l, b=(303.5,2.4)$ with a distance modulus of 12 mag $(2512 \mathrm{pc})$. In their new delineation of Galactic OB associations, Mel'nik $\&$ Efremov (1995) propose five groups (Cen 1A to Cen 1E) located between $303^{\circ} \leq l \leq 306^{\circ}$ at distances varying from 1.8 to $2.2 \mathrm{kpc}$. Their Cen 1A group consists of 37 stars, almost all belonging to NGC 4755. That group includes the 09.5type star HD 311999 seen toward the H II region Gum 46. The groups Cen 1B, Cen 1C, and Cen 1D consist of several field stars, while Cen 1E (6 stars) contains mostly members of the open cluster St 16. Recently, based on proper motion and spectrophotometric distances, Corti et al. (2012) have provided an updated list of 56 members of Cen OB1 at an average distance of $2.6 \pm 0.4 \mathrm{kpc}$. Figure 2 presents a comparison of the uvby $\beta$ distance moduli obtained here to previously published spectroscopic estimates (Humphreys 1978; Corti et al. 2012). The spectroscopic distance moduli appear to be larger as a result of overestimated intrinsic brightness in some of the existing spectroscopic calibrations (see for example Kaltcheva \& Golev 2011). The distance moduli for members of Cen OB1 selected by Corti et al. (2012) are all grouped around 12 mag as a consequence of the selection procedure adopted by the authors, which considered only stars with consistent proper motions located around the DM of 12 mag adopted by Humphreys (1978).

In the present study a homogeneous $u v b y \beta$ photometric distance scale was established for nearly 700 O-B9 field stars and the rich open cluster NGC 4755, permitting us to identify spatially coherent groups and layers. According to their derived distances and photometric quantities, there is a clear separation of intrinsically bright, massive, recently-born stars delineating relatively distant sites of star formation from nearby, less massive stars (Fig. 3). A tentative separation of sample stars is made at $M_{V}=-2 \mathrm{mag}$, which reflects their overall properties well. No clear interarm space is detected toward Centaurus, but there is instead a continuous distribution of stars between nearby Galactic features and the more distant Centaurus star forming complex (see Fig. 3, color excess vs. DM diagram). Interstellar extinction towards Centaurus is almost negligible up to $160 \mathrm{pc}$ $(\mathrm{DM}=6 \mathrm{mag})$. At $\sim 1 \mathrm{kpc}$ a steep increase in extinction is noticeable.

To search for coherent groups that can be connected to distant star forming features, we removed for the following analysis all stars with $M_{V}>-2$ mag. Figure 4 represents only stars with $M_{V}<-2$ mag, which are relatively distant with few exceptions. A group of very young stars is noticeable at about 1000 pc. Part of the group, concentrated toward average Galactic coordinates $l=305^{\circ}, b=1^{\circ}$, is quite reddened $\left(A_{V}=3 \mathrm{mag}\right)$. The remaining young stars located at $1000 \mathrm{pc}$ are less reddened and dispersed across the field. Since this is the first time these 40 stars have been distinguished, they are listed in Table 3, only available electronically. The format of the table follows the format of Table 1.

The rest of the intrinsically bright massive stars $\left(M_{V}<\right.$ $-2 \mathrm{mag}$ ) lie at distances of 1200 to $4000 \mathrm{pc}$ (see Fig. 4), delineating an extended field of recent star formation. Of them, 172 are located between 1200 and 3600 pc (median distance 1820 pc \pm 583 s.d.; \pm 44 s.e.). Eleven stars in Fig. 4 appear to be more distant than $\mathrm{DM}=13 \mathrm{mag}$.

We noticed that some of the very massive stars in the sample $\left(M_{V}<-5.5 \mathrm{mag}\right.$, not shown with separate symbols $)$ are detected towards $l=306^{\circ}$ probably tracing a tangential spiral segment in this direction. Longitude $l=308^{\circ}$ defines a tangential direction in the Galaxy. Distant OB stars towards $l=306^{\circ}$ have been previously noted by Jackson (1976). Turner (1985) confirmed the existence of (at least) four distant OB stars (LS 3031, LS 3033, LS 3035, and LS 3040) that are background to St 16. 
N. T. Kaltcheva et al.: Galactic supershell GSH 305+01-24
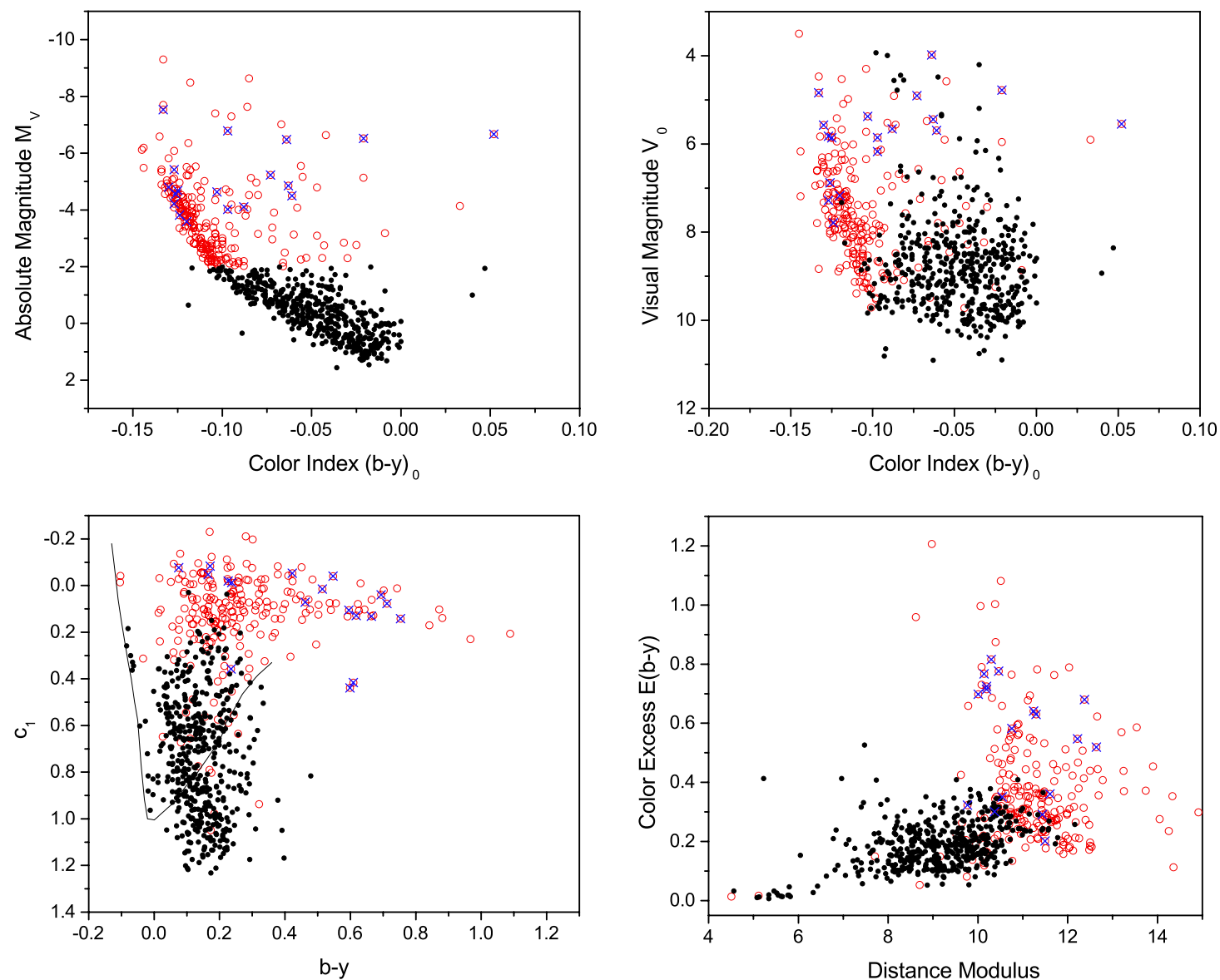

Fig. 3. Photometric diagrams $M_{V}$ vs. $(b-y)_{0}, V_{0}$ vs. $(b-y)_{0}$ and $c_{1}$ vs. $b-y$. The color excess $E(b-y)$ vs. DM is shown in the last panel. Symbols are the same as in Fig. 1: intrinsically bright stars with $M_{V}<-2$ mag are marked by red open circles, stars intrinsically fainter than -2 mag by black filled circles, stars from the classical Cen OB1 association by blue $\times$-symbols. See the electronic edition for a color version of the figure.
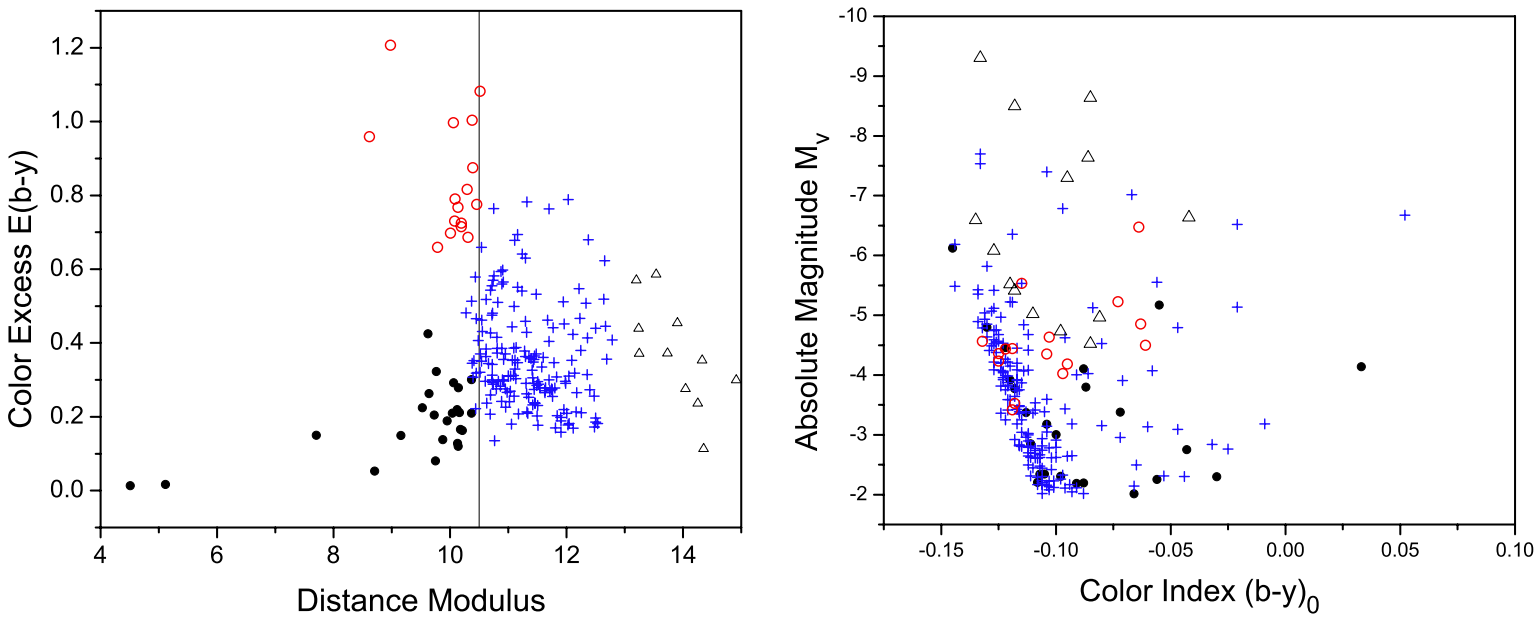

Fig. 4. $E(b-y)$ vs. DM and $M_{V}$ vs. $(b-y)_{0}$ diagrams for all stars intrinsically brighter than $M_{V}=-2$ mag. Open and filled circles represent stars at a distance around $1 \mathrm{kpc}$, blue cross symbols are for stars between 1 and $4 \mathrm{kpc}$, and triangles are for the most distant stars in the sample. See the electronic edition for a color version of the figure.

Complete $u v b y \beta$ photometry is available for one of the stars, yielding a distance modulus of $12.6 \mathrm{mag}$, in agreement with what is estimated by Turner (1985).

The three brightest members of St 16 are included in the uvby $\beta$ sample and yield a median distance modulus of $11.45 \pm$ $0.33 \mathrm{mag}$ and color excess $E(b-y)$ of $0.38 \pm 0.007(E(B-$ $V)=0.51)$. Both estimates agree with previous values based on $U B V$ photometry (see Turner 1985; Vázquez et al. 2005). A photometric distance modulus of $11.45 \mathrm{mag}(1950 \mathrm{pc})$ is found for the O7.5 III star HD 115455 (uvby98 431160111, the brightest cluster member), in agreement with the cluster distance estimates. The star is thought to be the exiting source for RCW 75 (see Vázquez et al. 2005, and references therein for details), which provides a reliable distance estimate for this H II region.

A new distance estimate is made for the cluster NGC 4755 based on 56 cluster members. The median and average values 


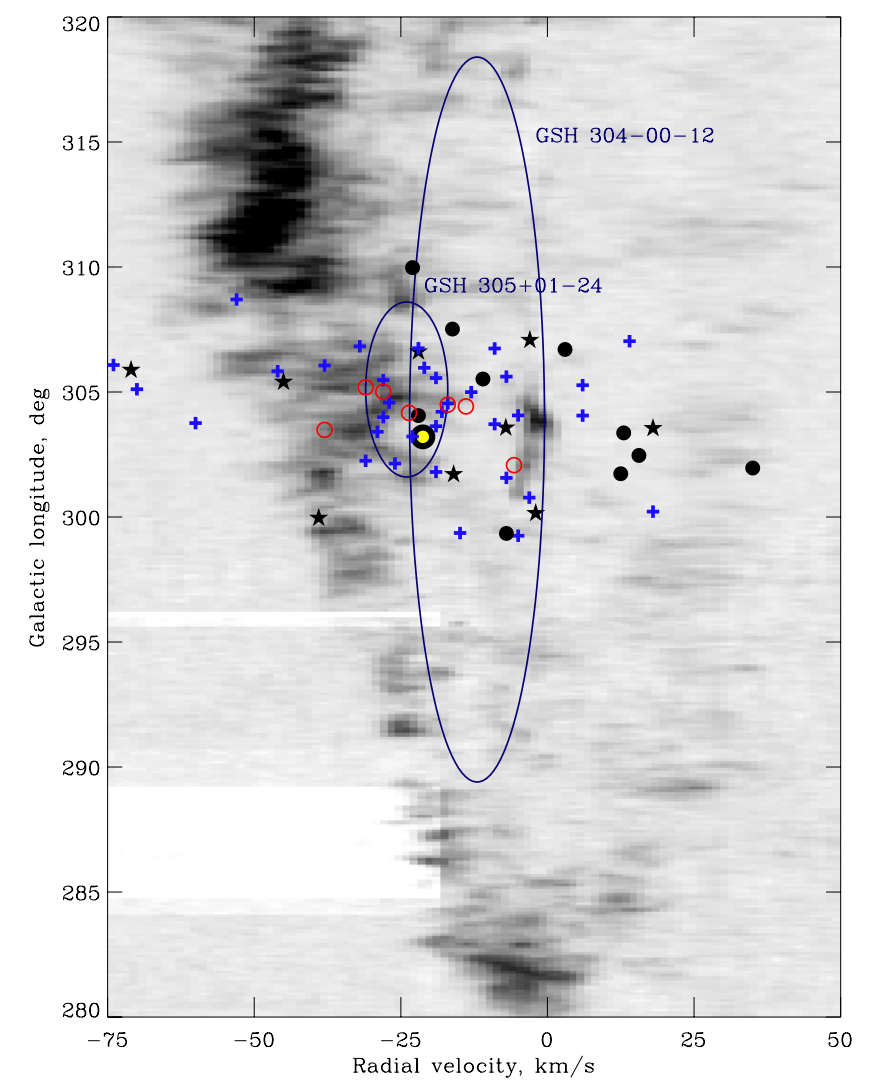

Fig. 5. Longitude-velocity map, ${ }^{12} \mathrm{CO} J=1-0(115 \mathrm{GHz})$ emission survey of Dame et al. (2001). GSH 304-00-12 and GSH 305+01-24 are marked by ellipses located at their cataloged positions whose sizes are determined by their angular diameters and velocity widths. As in Fig. 4, stars of high- and low-reddening at $1 \mathrm{kpc}$ are indicated by the red open and black filled circles, respectively, and blue cross symbols are for stars between 1 and $4 \mathrm{kpc}$. Intrinsically bright stars with $M_{V}<-5.5 \mathrm{mag}$ are shown with black 5-pointed star symbols. The position of NGC 4755 is shown with large yellow circle outlined with black. See the electronic edition for a color version of the figure.

for the sample are identical and yield a distance modulus of $11.48( \pm 0.46 \mathrm{sd} ; \pm 0.061 \mathrm{se})$. The corresponding mean distance is $1968 \pm 65 \mathrm{pc}$ and agrees closely with the average distance of the stars associated with the GSH305+01-24 shell (see Sect. 4). The average color excess is $E(b-y)=0.29( \pm 0.03 \mathrm{sd} ; \pm 0.0041 \mathrm{se})$. These estimates are in perfect agreement with other authors (see Corti \& Orellana 2013, and the reference therein).

In summary, the distances derived for nearly 230 massive field stars indicate that the Centaurus star-forming field is closer than estimated previously, at a median distance of $1.8 \mathrm{kpc}$ instead of the classical $2.5 \mathrm{kpc}$. This difference most likely arises from an overestimation of spectroscopic distances in previous studies. A portion of stars in the classical Cen OB1 belongs to the group detected at $1 \mathrm{kpc}$, while part is more distant. Turner (1985) provided a similar estimate of $1.88 \pm 0.24 \mathrm{kpc}$ to Cen OB1 based mainly on $\mathrm{H} \beta$ photometry of ten members of Cen OB1 with reliable luminosities. The partition of Cen OB1 into five groups by Mel'nik \& Efremov (1995) is not apparent. The overall impression is that beyond $1 \mathrm{kpc}$ an extended star-forming complex of many OB stars is located.

To shed more light on the groups and layers discussed above, we collected all available radial velocities for these stars. In Fig. 5, following McClure-Griffiths et al. (2002), we superimposed them on a longitude-velocity $(l, v)$ diagram based on the
${ }^{12} \mathrm{CO} J=1-0(115 \mathrm{GHz})$ datacube from the CO survey by Dame et al. (2001). The CO emission was averaged over the latitude range $|b|<2^{\circ}$, and the axis range was adjusted to include the Carina tangent at $l, v \sim 282^{\circ}, 0 \mathrm{~km} \mathrm{~s}^{-1}$ and the CruxScutum arm at $l, v \sim 310^{\circ},-50 \mathrm{~km} \mathrm{~s}^{-1}$. In the figure we show the foreground shell GSH 304-00-12 at a kinematic distance $1.2 \pm 0.8 \mathrm{kpc}$, also discovered by McClure-Griffiths et al. (2001).

As noted by McClure-Griffiths et al. (2002), the GSH 304-00-12 shell lies in the interarm region and is bounded by $\mathrm{CO}$ emission from the Coalsack nebula near $l, v \sim 301^{\circ}, 0 \mathrm{~km} \mathrm{~s}^{-1}$. GSH $305+01-24$ at $2.2 \pm 0.9 \mathrm{kpc}$ can be associated with the massive stars around $1.8 \mathrm{kpc}$, including NGC 4755. The highly-reddened group at $1 \mathrm{kpc}$ seems to be located on the near edge of the same shell, while the lowreddened layer at $1 \mathrm{kpc}$ seems to be kinematically connected to GSH 304-00-12.

\subsection{Comparison to other studies}

As noted earlier, several highly reddened stars from the 1-kpc layer, and also some of the most massive and distant stars in the sample are grouped toward $l, b=305^{\circ}, 1^{\circ}$. The region toward $l=305^{\circ}$ is particularly interesting because it contains the G305 H II complex (G305.4+0.1), one of the most massive starforming structures ever identified within the Galaxy (see Faimali et al. 2012), its central obscured young star clusters Danks 1 and Danks 2, the Wolf-Rayet stars WR 48a,b and $\theta$ Mus, other embedded clusters, several $\mathrm{H}$ II regions, $\mathrm{OH} / \mathrm{H}_{2} \mathrm{O}$ maser sources, and more (see Baume et al. 2009, for a detailed description).

Baume et al. (2009) present a wide-field photometric study of the Galactic plane toward $l=305^{\circ}$, revealing a significantly high interstellar absorption $\left(A_{V} \sim 10 \mathrm{mag}\right)$ and an abnormal extinction law in the line of sight. They found (see also Vázquez et al. 2005; and Carraro \& Costa 2009) three different spiral features at increasing distances from the Sun: a spatially spread foreground population placed mainly between $350-750 \mathrm{pc}$, a second feature situated just behind a very dark cloud at $1-3 \mathrm{kpc}$ and represented by the clusters Danks 1 and 2, and finally a third population that is traced by embedded clusters located at about 5-7 kpc.

These results are in accord with our findings for the field. In our sample, the spatially spread foreground population is represented by stars intrinsically fainter than -2 mag (see Fig. 3). The highly reddened stars at $1 \mathrm{kpc}$ are located at the near end of the dust cloud seen toward $l=305^{\circ}$, at the near edge of the Carina arm. The Centaurus complex stretches through the arm, approximately between $1.2 \mathrm{kpc}$ and $3.3 \mathrm{kpc}$. The distant, low-reddened stars we have in our sample lie between $4 \mathrm{kpc}$ and $6.5 \mathrm{kpc}$, consistent with the near end of the Scutum-Crux arm.

It can be noted that the uncertainties in distance to both embedded clusters and ISM features toward the Centaurus-Crux tangent are quite large. Recently Davies et al. (2012) have found a kinematic distance to G305 of $4.2 \pm 2.0 \mathrm{kpc}$ and a spectrophotometric distance to Danks 1 and 2 of $3.48_{-0.71}^{+0.91} \mathrm{kpc}$ and $2.61_{-0.61}^{+0.82} \mathrm{kpc}$, respectively. By comparison, the $u v b y \beta$ distances delineate at least the near side of the Centaurus star-forming field more precisely.

\section{Census of OB stars within GSH $305+01-24$}

As already noted, the massive, intrinsically luminous stars found between $1 \mathrm{kpc}$ and $4 \mathrm{kpc}$ delineate an extended star-forming field. Of this sample, 133 stars are projected over the sky area 

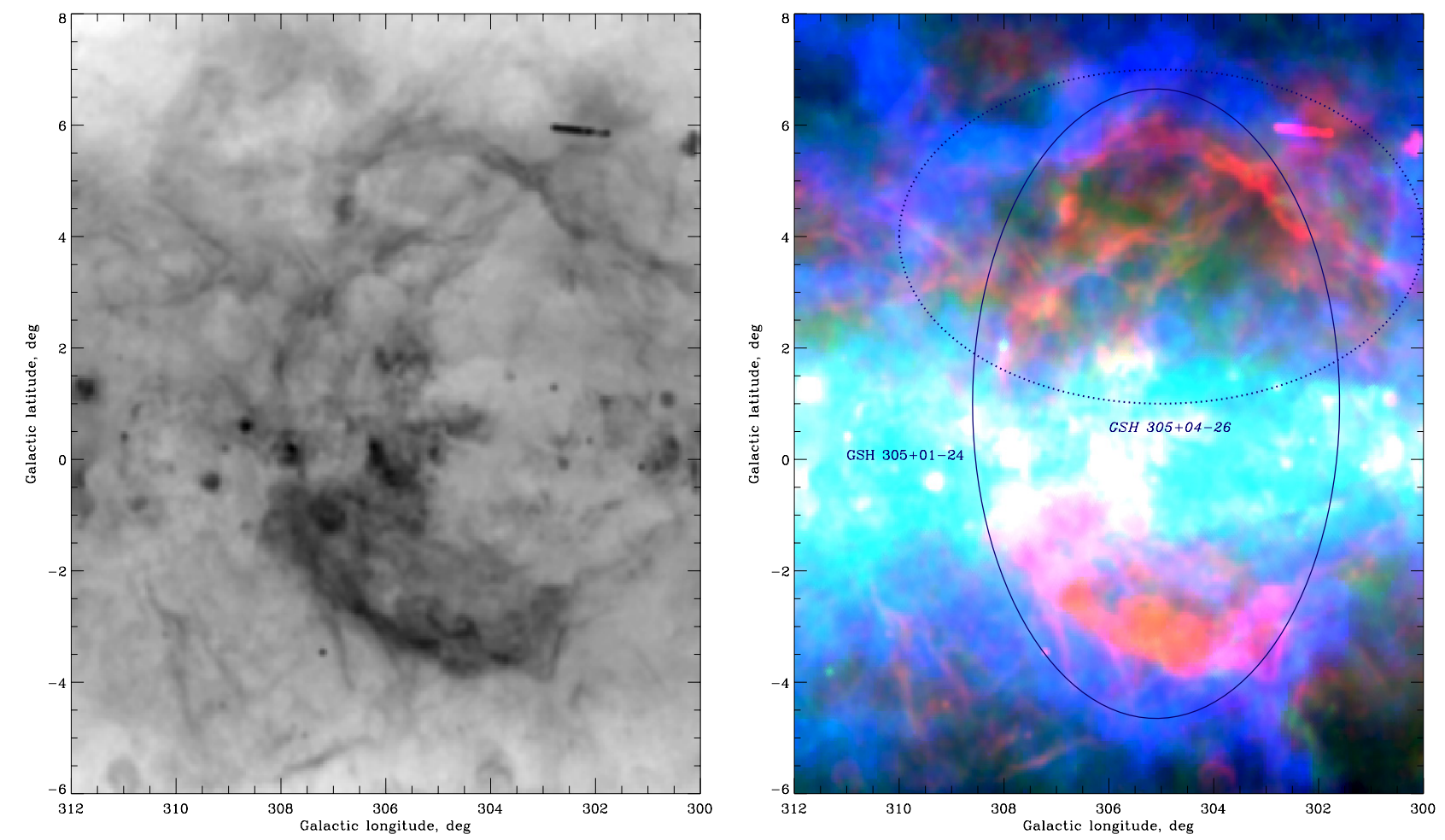

Fig. 6. Left: $\mathrm{H} \alpha$ emission (data by Finkbeiner 2003) toward the Coalsack Loop. Right: $\mathrm{H} \alpha$ emission (red) combined with $\mathrm{H}$ I 21 -cm emission (blue) and $100 \mu \mathrm{m}$ DIRBE far-IR emission (green) for the same region. The solid-line ellipse outlines the H I shell GSH 305+01-24 discovered by McClure-Griffiths et al. (2001). The H I 3D-datacube of the region were taken from The Southern Galactic Plane Survey (McClure-Griffiths et al. 2005). Here the $-24 \mathrm{~km} \mathrm{~s}^{-1}$ channel of $\mathrm{H}$ I emission is shown. The position of the shell GSH 305+04-26 (labeled in italics) recently proposed by Corti et al. (2012) is outlined by a dotted line. See text for discussion. See the electronic edition for a color version of the figure.

covered by GSH $305+01-24$ and are located at an average distance of $1.8 \pm 0.4 \mathrm{kpc}$ (mean $\mathrm{DM}=11.3 \pm 0.60$, median $\mathrm{DM}=11.24)$. We consider this "shell subsample" as part of the massive stellar population of GSH 305+01-24 that matters from the wind injection energy viewpoint.

To estimate the completeness of this shell subsample, we first compared the selection to the HIPPARCos catalog. Only five stars were found there, all of them with very small or negative parallaxes, as should be expected for stars more distant than $1 \mathrm{kpc}$. This confirms our statement that the shell subsample is not contaminated by any nearby young stars. This is especially important for the Centaurus star-forming field, which is background to the nearby Sco-Cen association.

Nearly $70 \%$ of the shell subsample stars have HD numbers as primary designations. Because of some spectral type discrepancies in the B8-A2 range that appear in the HD catalog, it is difficult to estimate the completeness precisely. For example, HD 112537 is classified as B9 in the HD catalog, but as A1V in SIMBAD and thus omitted in our sample which is focused on stars of spectral types earlier than A0. An estimate reflecting these discrepancies indicates that the shell subsample is $\sim 90 \%$ complete to the magnitude limit of the HD catalog. A similar estimate was obtained for the completeness of stars having only $\mathrm{BD} / \mathrm{CD} / \mathrm{CPD}$ identifiers. These estimates point out that the completeness of the sample within the shell is about $85-90 \%$ to a limiting magnitude of 11.5-12 mag for spectral types O-B9.

\subsection{Observational properties of the GSH 305+01-24 shell}

GSH 305+01-24 was first recognized as a large ring of $\mathrm{H} \alpha$ emitting nebulosity of about $10^{\circ}$ in diameter, surrounding the
Coalsack Nebula and was referred to as "The Coalsack Loop" (Walker \& Zealey 1998, see our Fig. 6 left). Based on observations with the Parkes Radio Telescope, it was then identified with a large H I supershell in this direction (McClure-Griffiths et al. 2001). The parameters of GSH 305+01-24 are summarized by McClure-Griffiths et al. (2002) as follows: $l, b=305^{\circ} 1,+1^{\circ} .0$; $\Delta l \times \Delta b=7^{\circ} \times 11^{\circ} .3$; central radial velocity $v_{\mathrm{LSR}}=-24 \mathrm{~km} \mathrm{~s}^{-1}$; velocity full width $\Delta v=14 \mathrm{~km} \mathrm{~s}^{-1}$, and expansion velocity $v_{\text {exp }} \sim 7 \mathrm{~km} \mathrm{~s}^{-1}$. The shell extends between $-5^{\circ}$ and $+7^{\circ}$ across the Galactic plane. According to McClure-Griffiths et al. (2001), $v_{\mathrm{LSR}}=-24 \mathrm{~km} \mathrm{~s}^{-1}$ implies a kinematic distance of $2.2 \pm 0.9 \mathrm{kpc}$ and physical dimensions of $280 \times 440 \mathrm{pc}$. Since the estimated distance of GSH $305+01-24$ at $2.2 \mathrm{kpc}$ is consistent with that of Cen OB1 at $2.5 \mathrm{kpc}$, as determined by Humphreys (1978), the H I shell has been identified as blown out by the classical Cen OB1 association. However, the kinematic distance of the shell is just as consistent with the revised distance to Cen OB1.

The distance to the shell subsample obtained here places the shell at a photometric distance of $1.8 \pm 0.4 \mathrm{kpc}$. Within the errors, this estimate agrees with the kinematic distance $2.2 \pm 0.9 \mathrm{kpc}$ and implies a shell size $a \times b=229 \times 360 \mathrm{pc}$. The harmonic equivalent radius is calculated as $R_{\mathrm{s}}=0.5 \sqrt{a \times b} \approx$ $143.5 \pm 32 \mathrm{pc}$.

Figure 6 (right) presents the HI radio data obtained from the archive of The Southern Galactic Plane Survey (McClure-Griffiths et al. 2005) combined with the $\mathrm{H} \alpha$ data by Finkbeiner (2003) and $100 \mu \mathrm{m}$ DIRBE far-IR data (Schlegel et al. 1998). The GSH 305+01-24 is outlined. The Coalsack Loop shows a striking similarity between the $\mathrm{H} \alpha$ (inside) and $\mathrm{HI}$ (outside) emission morphologies. In Fig. 6 (right) the GSH 305+04-26 shell, that was recently suggested 
by Corti et al. (2012), is also shown. Based on H I data, these authors argue that the two halves of GSH 305+01-24, located above and below the Galactic plane (their features A and B), are unrelated H I structures and do not manifest different parts of the same feature, as suggested by McClure-Griffiths et al. (2001). The $\mathrm{H} \alpha$ image, however, does not support this conclusion. The ISM emissions at different wavelengths and the distribution of the stellar content of the shell confirm the existence of only one shell, with $\mathrm{H}$ I outward, $\mathrm{H} \alpha$ inward, and a cavity full of dust IR emission. The finding of Corti et al. (2012) of two unrelated H I shells above and below the Galactic plane is not confirmed by the present results.

\subsection{Wind-blown energy deposit of the most massive stars within GSH 305+01-24}

Ionized shells around OB associations are usually connected to the initial stages of star cluster blown bubbles (e.g. Silich et al. 2008; Silich \& Tenorio-Tagle 2013) and are suggested as progenitors of the larger H I supershells (see Relaño et al. 2007; Silich \& Tenorio-Tagle 2013). The mechanical luminosities of the stellar winds of massive MS stars influence the size and shape of the HI superbubbles. The final size of a superbubble created in a molecular environment during the massive stars MS stage depends on the stellar mass loss, wind velocity, and environmental density. Since stars in the mass range 8 to $25-30 M_{\odot}$ will end their lives in an RSG phase, the extent of molecular gas cavity is largely determined by the merged wind bubbles (i.e., combined bubble blown from the contribution of the output of all massive stars).

The energetics of the H I shells are usually characterized by the expansion energy, $E_{\text {exp }}$, defined as the equivalent energy instantaneously deposited at the shell center to account for the shells radius, $R_{\mathrm{s}}$, and also by the current rate of expansion, $v_{\text {exp }}$. As in McClure-Griffiths et al. (2001), to estimate the expansion energy of GSH 305+01-24, we used the expression (Chevalier 1974; Heiles 1979):

$E_{\text {exp }}=5.3 \times 10^{+43} n_{0}^{1.12} R_{\mathrm{s}}^{3.12} v_{\text {exp }}^{1.4} \approx(3.5 \pm 1.9) \times 10^{51} \mathrm{erg}$,

where the ambient density $n_{0}$ is adopted to be $1 \mathrm{~cm}^{-3}$, a typical value (see e.g. Lozinskaya 1999, for details).

In the absence of an observed power source, the real age of the shell could not be determined. Instead, an age estimate based on dynamic properties, or the dynamic age, can be quoted. Following Corti et al. (2012), who used the Weaver et al. (1977) standard model for a thin expanding shell created by a continuous injection of mechanical energy, we derive the dynamic age $t_{\text {dyn }}$ of GSH 305+01-24 as:

$t_{\text {dyn }}=0.55 \times 10^{6} R_{\mathrm{s}} / v_{\exp } \approx 13.15 \pm 2.95 \mathrm{Myr}$.

Next we calculate whether the stars with masses $M \gtrsim 8 M_{\odot}$ within the shell are able to contribute a sufficient wind injection energy in order to explain the observed size and expansion velocity. Figure 7 presents the HR diagram for the shell subsample with masses $M \gtrsim 8 M_{\odot}$. NGC 4755 is also included. The effective temperatures $T_{\text {eff }}$ and bolometric corrections are obtained via calibrations in terms of $c_{0}$ and $[u-b]$ (see Davis \& Shobbrook 1977; Code et al. 1976). Evolutionary tracks (mass range $6 \div 45 M_{\odot}$ ) and isochrones (log age range $6.0 \div 7.9$ ) by Ekström et al. (2012) extracted via the Geneva stellar models: interactive tools ${ }^{1}$ are overplotted. We use models

\footnotetext{
1 http://obswww. unige.ch/Recherche/evoldb/index/
}

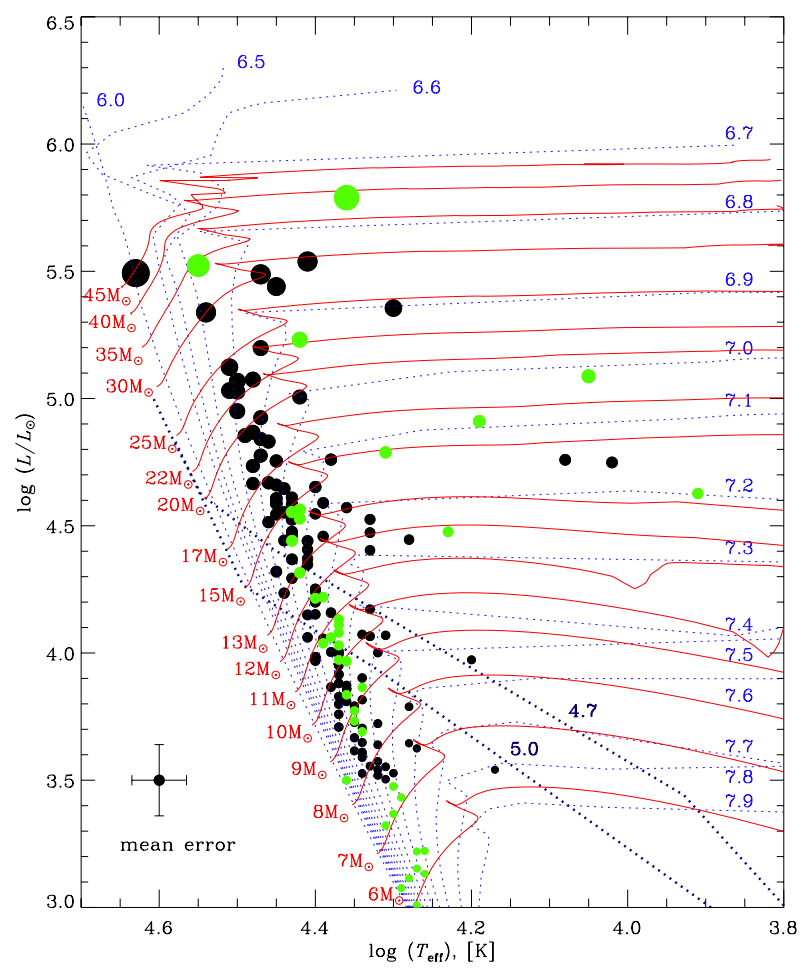

Fig. 7. HR diagram for the stellar content of GSH 05+01-24. Stars of different masses are represented with filled circles of different diameters. Black circles are used for the shell subsample, and green circles denote NGC 4755 massive members. See the text for details. See the electronic edition for a color version of the figure.

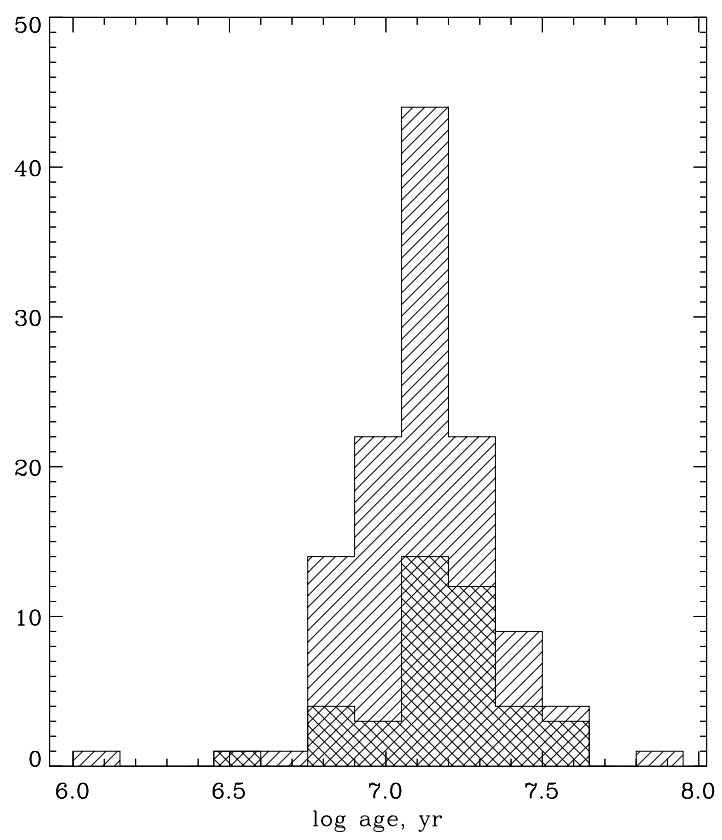

Fig. 8. Histogram of stellar ages for the shell subsample (119 stars, diagonal pattern) and NGC 4755 sample (41 stars, cross-lined pattern).

with initial metallicity $Z_{\text {init }}=0.014$ (solar) and initial rotation rate $v / v_{\text {crit }}=0.40$. Two isochrones for pre-main sequence stage (log ages 4.7 and 5.0, Claret 2012) are also shown. The individual stellar ages are extracted from the models. The individual log age distributions for the shell subsample and NGC 4755 sample are shown in Fig. 8. We estimate an average age of the shell subsample of $7.087 \pm 0.177$ or $\approx 12.2 \pm 4.09 \mathrm{Myr}$, in good agreement with the dynamic age $t_{\text {dyn }}$ of the shell $(13.15 \pm 2.95 \mathrm{Myr})$, 

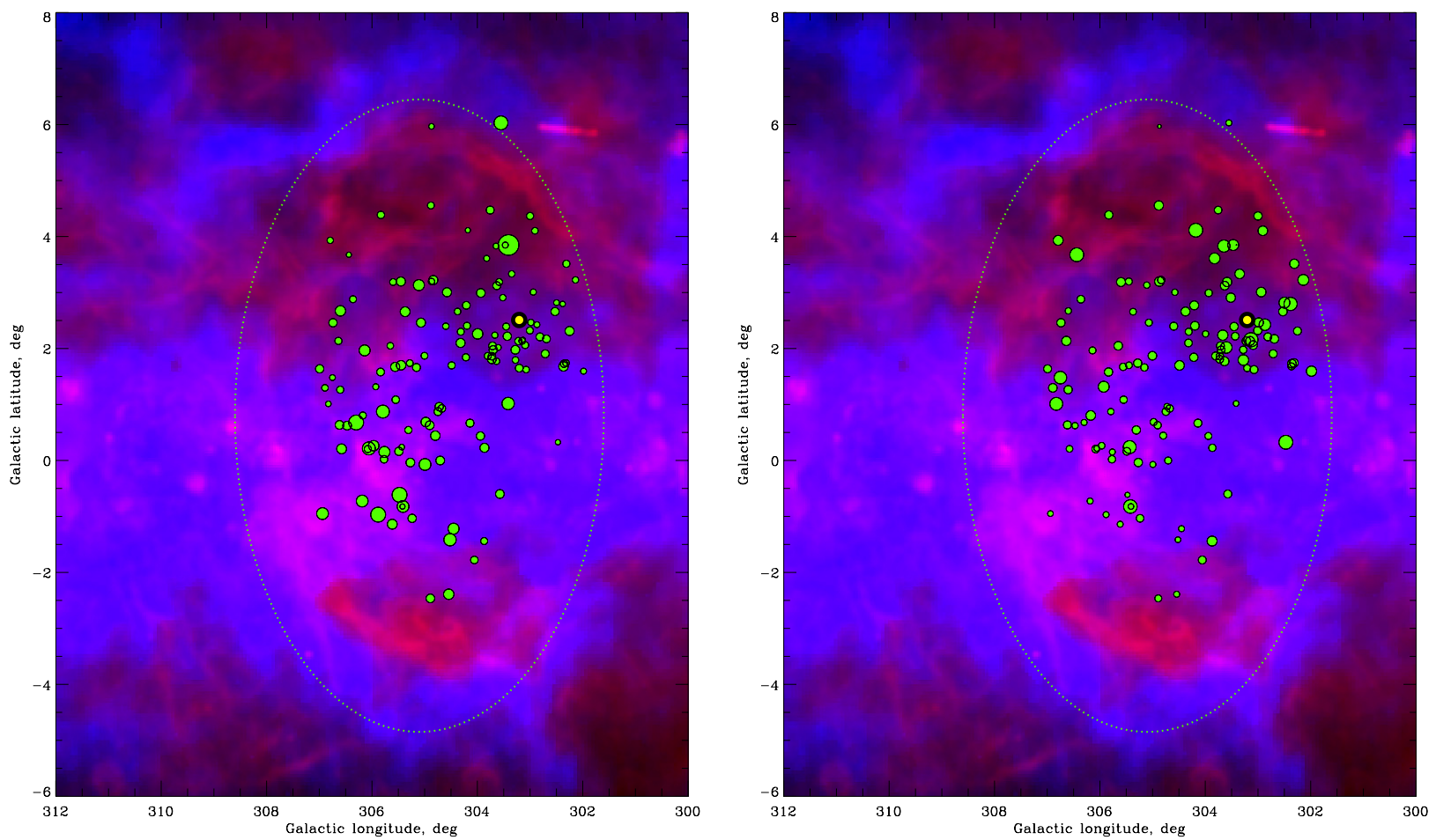

Fig. 9. Massive stars within the Coalsack Loop. $\mathrm{H} \alpha$ emission is shown in red and H I $21-\mathrm{cm}$ emission $\left(-24 \mathrm{~km} \mathrm{~s}^{-1}\right.$ channel) is in blue. Left: symbol size is proportional to the stellar mass. Right: symbol size is proportional to the stellar age. The position of NGC 4755 is shown with a yellow circle outlined with black. See the electronic edition for a color version of the figure.

calculated above. For NGC 4755 the average log age derived by us is $7.183 \pm 0.230$ or $15.2 \mathrm{Myr}$.

The estimates for the distance and age of NGC 4755 that we derived are in very good agreement with previous results. Corti \& Orellana (2013) give the distance to the cluster as $1.9 \pm 0.5 \mathrm{kpc}$ and the age of $\sim 15$ Myr. Bonatto et al. (2006) estimated the cluster's age as $14 \pm 2 \mathrm{Myr}$ and concluded that the star formation in NGC 4755 began $\approx 14$ Myr ago and proceeded for about the same length of time. Lyra et al. (2006) find a value of $2040 \pm 250 \mathrm{pc}$ for the distance to the cluster and $7.1 \pm 0.2$ for the log age. WEBDA database ${ }^{2}$ lists log age 7.216 or $16.4 \mathrm{Myr}$ and distance $1976 \mathrm{kpc}$.

One of the main results of this work is that NGC 4755 is practically at the same distance as the shell subsample. The mean radial velocity of NGC $4755\left(-21.24 \pm 0.89 \mathrm{~km} \mathrm{~s}^{-1}\right.$, Mermilliod et al. 2008) coincides well with the mean radial velocity of GSH 305+01-24. These are firm arguments that NGC 4755 and the shell subsample form the main part of the massive star population within the shell that should be taken into account when studying the energetics and star-formation history of this region.

In Fig. 9 all stars included in the HR diagram (hence with calculated masses and ages) are plotted against the Coalsack Loop. As in Fig. 6, the $\mathrm{H} \alpha$ emission is combined with H I 21-cm emission $\left(-24 \mathrm{~km} \mathrm{~s}^{-1}\right.$ channel). In the left hand panel, the stars are represented with symbols proportional to their mass as estimated from the Geneva stellar models. In the right hand panel, the symbol size is proportional to star's estimated age. One can follow an age gradient over the Coalsack Loop with NGC 4755 the oldest. Continuous star formation might be taking place within the shell with the youngest, most massive stars located toward the periphery of the shell. The stars appear to trace the high $\mathrm{H} \alpha$ emission, avoiding it at the same time. The projected

\footnotetext{
2 http://www. univie.ac.at/webda/Welcome.html
}

coincidence of the OB stars with the shell and the similarities between the shells morphology and the OB-star distribution indicate a strong interaction of the stellar winds with the superbubble material, as also suggested by McClure-Griffiths et al. (2001).

The kinetic energy deposit into the surrounding media can be estimated based on stellar wind luminosities and stellar ages. Only stars with masses $M \gtrsim 8 M_{\odot}$ were taken into account (113 stars from the shell subsample and 30 member stars of NGC 4755). The parameters involved in the calculations are the wind mass-loss rate, $\dot{M}$, which is the amount of mass lost by the star per unit time, and the terminal (asymptotic) velocity $v_{\text {inf }}$ or the velocity of the stellar wind at a large distance from the star. The $\log$ values of $\dot{M}$ in units $\left[M_{\odot} \mathrm{yr}^{-1}\right]$ were obtained from the models. The wind terminal velocity can be estimated from Leitherer et al. (1992, Eq. (2)) as a function of luminosity, mass, and $T_{\text {eff }}$ of the star (solar metallicity was adopted). With these parameters in hand, the wind energy production of each star during its MS lifetime, $E$, was estimated as

$E=\frac{1}{2} \dot{M} v_{\mathrm{inf}}^{2} \tau_{\mathrm{ms}}$

(see Eq. (1) in Chevalier 1999) where $\tau_{\mathrm{ms}}$ is the main-sequence age of the star, obtained from the models. Thus the integrated wind energy deposit of all stars was found to be $E_{\text {wind }} \approx 2.95 \times$ $10^{51} \mathrm{erg}$, in quite good agreement with shell's expansion energy $E_{\exp } \approx(3.5 \pm 1.9) \times 10^{51}$ erg calculated above.

Ionized shells may present the inner skins of neutral shells if the number of ionizing photons emerging from the central cluster is not sufficient to ionize the whole shell. It looks like the observed shell is passing through this stage at the present time.

Using a basic model in which energy is supplied first by stellar winds and then by supernovae, Relaño et al. (2007) extrapolated the properties of these shells in time, finding results in good 
overall agreement with the observed properties of the $\mathrm{H}$ I shells after times of a few $10^{7} \mathrm{yr}$. They also discuss that, if the formation of the OB stars occurs on shorter time scales, the stars will no longer be located at the centers of the H I shells (see also Hatzidimitriou et al. 2005, observations reported for the SMC). Our results appear to support that. However, although the massive stellar content of GSH 305+01-24 appears shifted towards the near edge of the shell, the large uncertainties in the shell kinematic distances do not allow firm conclusions.

\section{Concluding remarks}

The $\mathrm{H} \alpha, \mathrm{HI},{ }^{12} \mathrm{CO}$, and $100 \mu \mathrm{m}$ emission in the direction of the Centaurus star-forming field was used to explore the morphology of the Galactic supershell GSH 305+01-24. A superposition of large-scale features of the Milky Way disk and the supershell GSH 305+01-24, which extends perpendicularly to the disk, were clearly outlined. The morphology of $\mathrm{H} \alpha$ and $\mathrm{H}$ I features are consistent with the location of GSH 305+01-24 as delineated by McClure-Griffiths et al. (2001). The positional coincidence of OB stars and the shell, along with similarities between the shell's morphology and the distribution of OB stars, indicates a strong interaction of the stellar winds and the bubble material. The precise photometric distances obtained here revealed the presence of previously unrecognized groups of stars, and led to a complete revision of the OB-star distribution in the Centaurus star-forming complex. The young stellar component beyond $1 \mathrm{kpc}$ occupies the cavity of an extended $\mathrm{HII}$ region that is surrounded by the HI GSH 305+01-24 supershell. We identified this component at about $85-90 \%$ completeness up to 11.5-12 mag. We show that these stars are able to contribute enough wind injection energy to explain the observed size and expansion velocity. A previously undetected layer of very young stars was also identified at $1 \mathrm{kpc}$, and its connection to both GSH 305+01-24 and 304-00-12 H I shells was investigated.

Acknowledgements. This work is supported by the National Science Foundation grant AST-0708950. N.K. acknowledges support from the SNC Endowed Professorship at the University of Wisconsin Oshkosh. K.M. acknowledges support from the Wisconsin Space Grant Consortium. V.G. acknowledges support by the Bulgarian National Science Research Fund under grant DO 02$85 / 2008$. This study made use of the NASA Astrophysics Data System, SIMBAD database, Centre de Données Stellaires ${ }^{3}$, WEBDA open cluster database operated at the Institute for Astronomy of the University of Vienna, and NASA's SkyView facility ${ }^{4}$ located at NASA Goddard Space Flight Center (see McGlynn et al. 1998). We acknowledge the use of the Southern H $\alpha$ Sky Survey Atlas (SHASSA), which is supported by the National Science Foundation (Gaustad et al. 2001). We acknowledge the use of The Southern Galactic Plane Survey (see McClure-Griffiths et al. 2005). We are grateful to Sergiy Silich and David Turner for numerous helpful comments. We are thankful to the referee, Dr F. Comerón, for many valuable comments that greatly improved the paper.

\section{References}

Árnadéttir, A. S., Feltzing, S., \& Lundström, I. 2010, A\&A, 521, A40 Balona, L. A. 1994, MNRAS, 268, 119

Balona, L. A., \& Shobbrook, R. R. 1984, MNRAS, 211, 375

Baume, G., Carraro, G., \& Momany, Y. 2009, MNRAS, 398, 221

Benjamin, R. A. 2008, in Massive Star Formation: Observations Confront

Theory, eds. H. Beuther, H. Linz, \& T. Henning, ASP Conf. Ser., 387, 375

Blaha, C., \& Humphreys, R. M. 1989, AJ, 98, 1598

Bloemen, J. B. G. M., Deul, E. R., \& Thaddeus, P. 1990, A\&A, 233, 437

Bonatto, C., Bica, E., Ortolani, S., \& Barbuy, B. 2006, A\&A, 453, 121

Bronfman, L. 1992, in The Center, Bulge, and Disk of the Milky Way, ed.

L. Blitz, Astrophys. Space Sci. Lib., 180, 131

\footnotetext{
3 http://cdsweb.u-strasbg.fr/

4 http://skyview.gsfc.nasa.gov/
}

Carraro, G., \& Costa, E. 2009, A\&A, 493, 71

Chevalier, R. A. 1974, ApJ, 188, 501

Chevalier, R. A. 1999, ApJ, 511, 798

Claret, A. 2012, A\&A, 541, A113

Code, A. D., Bless, R. C., Davis, J., \& Brown, R. H. 1976, ApJ, 203, 417

Comerón, F. 2001, A\&A, 365, 417

Corti, M. A., \& Orellana, R. B. 2013, A\&A, 553, A108

Corti, M. A., Arnal, E. M., \& Orellana, R. B. 2012, A\&A, 546, A62

Crawford, D. L. 1978, AJ, 83, 48

Dame, T. M., Hartmann, D., \& Thaddeus, P. 2001, ApJ, 547, 792

Davies, B., Clark, J. S., Trombley, C., et al. 2012, MNRAS, 419, 1871

Davis, J., \& Shobbrook, R. R. 1977, MNRAS, 178, 651

de Zeeuw, P. T., Hoogerwerf, R., de Bruijne, J. H. J., Brown, A. G. A., \& Blaauw, A. 1999, AJ, 117, 354

Ekström, S., Georgy, C., Eggenberger, P., et al. 2012, A\&A, 537, A146

Elias, F., Alfaro, E. J., \& Cabrera-Caño, J. 2009, MNRAS, 397, 2

Faimali, A., Thompson, M. A., Hindson, L., et al. 2012, MNRAS, 426, 402

Finkbeiner, D. P. 2003, ApJS, 146, 407

Garmany, C. D., \& Stencel, R. E. 1992, A\&AS, 94, 211

Gaustad, J. E., McCullough, P. R., Rosing, W., \& Van Buren, D. 2001, PASP, 113,1326

Hatzidimitriou, D., Stanimirovic, S., Maragoudaki, F., et al. 2005, MNRAS, 360, 1171

Hauck, B., \& Mermilliod, M. 1998, A\&AS, 129, 431

Heiles, C. 1979, ApJ, 229, 533

Humphreys, R. M. 1978, ApJS, 38, 309

Humphreys, R. M., \& Kerr, F. J. 1974, ApJ, 194, 301

Jackson, P. D. 1976, Ph.D. Thesis, Maryland Univ., College Park

Kaltcheva, N. 2009, PASP, 121, 1045

Kaltcheva, N. T., \& Georgiev, L. N. 1994, MNRAS, 269, 289

Kaltcheva, N., \& Golev, V. 2011, in Stellar Clusters and Associations: A RIA Workshop on Gaia, 299

Kaltcheva, N. T., \& Golev, V. K. 2012, PASP, 124, 128

Kaltcheva, N. T., \& Hilditch, R. W. 2000, MNRAS, 312, 753

Kaltcheva, N., \& Makarov, V. 2007, ApJ, 667, L155

Kaltcheva, N. T., Olsen, E. H., \& Clausen, J. V. 2000, A\&AS, 146, 365

Kilkenny, D., \& Whittet, D. C. B. 1985, MNRAS, 216, 127

Lépine, J. R. D., Mishurov, Y. N., \& Dedikov, S. Y. 2001, ApJ, 546, 234

Leitherer, C., Robert, C., \& Drissen, L. 1992, ApJ, 401, 596

Lozinskaya, T. A. 1999, in New Perspectives on the Interstellar Medium, eds. A. R. Taylor, T. L. Landecker, \& G. Joncas, ASP Conf. Ser., 168, 427

Lyra, W., Moitinho, A., van der Bliek, N. S., \& Alves, J. 2006, A\&A, 453, 101

McClure-Griffiths, N. M., Dickey, J. M., Gaensler, B. M., \& Green, A. J. 2001, ApJ, 562, 424

McClure-Griffiths, N. M., Dickey, J. M., Gaensler, B. M., \& Green, A. J. 2002, ApJ, 578, 176

McClure-Griffiths, N. M., Dickey, J. M., Gaensler, B. M., et al. 2005, ApJS, 158, 178

McGlynn, T., Scollick, K., \& White, N. 1998, in New Horizons from Multi-Wavelength Sky Surveys, eds. B. J. McLean, D. A. Golombek, J. J. E. Hayes, \& H. E. Payne, IAU Symp., 179, 465

Mel'nik, A. M., \& Efremov, Y. N. 1995, Astron. Lett., 21, 10

Mermilliod, J. C., Mayor, M., \& Udry, S. 2008, A\&A, 485, 303

Rautiainen, P., \& Mel'nik, A. M. 2010, A\&A, 519, A70

Reed, B. C. 2003, AJ, 125, 2531

Relaño, M., Beckman, J. E., Daigle, O., \& Carignan, C. 2007, A\&A, 467, 1117

Russeil, D. 2003, A\&A, 397, 133

Sartori, M. J., Lépine, J. R. D., \& Dias, W. S. 2003, A\&A, 404, 913

Schlegel, D. J., Finkbeiner, D. P., \& Davis, M. 1998, ApJ, 500, 525

Silich, S., \& Tenorio-Tagle, G. 2013, ApJ, 765, 43

Silich, S., Elias, F., \& Franco, J. 2008, ApJ, 681, 1327

Stephenson, C. B., \& Sanduleak, N. 1971, Publications of the Warner \& Swasey Observatory, 1,1

Strömgren, B. 1966, ARA\&A, 4, 433

Taylor, J. H., \& Cordes, J. M. 1993, ApJ, 411, 674

Torra, J., Fernández, D., Figueras, F., \& Comerón, F. 2000, Ap\&SS, 272, 109

Turner, D. G. 1985, ApJ, 292, 148

Vázquez, R. A., Baume, G. L., Feinstein, C., Nuñez, J. A., \& Vergne, M. M. 2005, A\&A, 435, 883

Vallée, J. P. 2008, AJ, 135, 1301

Walker, A., \& Zealey, W. J. 1998, PASA, 15, 79

Weaver, R., McCray, R., Castor, J., Shapiro, P., \& Moore, R. 1977, ApJ, 218, 377

Zhang, E.-H. 1983, AJ, 88, 825 